\title{
Impacto de um programa de intervenção nutricional com idosos portadores de doença renal crônica
}

\author{
The impact of a nutrition intervention program \\ targeting elderly people with chronic kidney disease
}

Fernanda Guilhermino Magalhães ${ }^{1}$

Rita Maria Monteiro Goulart ${ }^{1}$

Leandro Campi Prearo ${ }^{2}$

${ }^{1}$ Universidade São Judas Tadeu. R. Taquari 546, Mooca. 03166-000 São Paulo SP Brasil. fmagalhaes@saojudas.br ${ }^{2}$ Pró-Reitoria de PósGraduação e Pesquisa, Universidade Municipal de São Caetano do Sul. São Caetano do Sul SP Brasil.
Abstract Chronic kidney disease (CKD) is recognized as a complex disease that requires multiple approaches to its treatment. The aim of this study was to evaluate the impact of a nutritional intervention program on the nutritional status and quality of life of elderly pre-dialysis CKD patients. A prospective cohort study was carried out involving 64 elderly stage 3 CKD patients receiving treatment at a Primary Care Center in the Municipality of Diadema in the State of São Paulo, Brazil. The nutritional intervention consisted of one individual and three group meetings. Nutritional status was assessed using anthropometric variables and classified according to Body Mass Index (BMI). Cardiovascular risk was classified according to Waist Circumference (WC). Quality of life was assessed using the WHOQOL-BREF. The data was analyzed adopting a significance level of $5 \%$. Mean age was $73.95 \pm 7.84$ years and the majority of the sample were women, had a low level of schooling, and low monthly income. With respect to nutritional status, $21.9 \%$ of the sample were underweight, $32.8 \%$ overweight, and $62.6 \%$ were at a high or very high risk of developing cardiovascular disease. The nutritional intervention program had a positive impact on nutritional status, leading to a decline in BMI and WC, reduction in risk of developing heart disease, increased satisfaction with current state of health, and improved quality of life. Key words Chronic Kidney Disease. Aging. Food and Nutrition Education
Resumo A doença renal crônica (DRC) é complexa, exigindo múltiplas abordagens em seu tratamento. O objetivo do presente estudo foi avaliar o impacto de um programa de intervenção nutricional sobre o estado nutricional e a qualidade de vida de idosos com DRC não dialítica. Foi realizada uma coorte prospectiva com 64 idosos, de ambos os sexos, com DRC estágio 3, atendidos em Unidade Básica de Saúde, em Diadema/SP. A intervenção nutricional consistiu em três encontros coletivos e um individual. O estado nutricional foi avaliado por antropometria, classificado pelo Indice de Massa Corporal (IMC) e o risco cardiovascular pela circunferência da cintura (CC). A qualidade de vida foi avaliada pelo WHOQOL -bref. Para as análises estatísticas, adotou-se nível de significância de 5\%. A amostra apresentou idade média de 73,95 \pm 7,84 anos, sendo a maioria do sexo feminino. Quanto ao estado nutricional, 21,9\% apresentou baixo peso, 32,8\% excesso de peso e 62,6\% risco cardiovascular elevado ou muito elevado. O programa de intervenção proporcionou impacto positivo no estado nutricional, com redução do IMC e da CC, diminuindo o risco de desenvolvimento de doenças cardiovasculares e promoveu aumento da satisfação dos idosos em relação ao estado de saúde repercutindo na melhora da qualidade de vida.

Palavras-chave Insuficiência renal crônica, Envelhecimento, Educação alimentar e nutricional 


\section{Introdução}

A doença renal crônica (DRC), definida como a presença de anormalidades na estrutura ou na função renal por um período igual ou superior a três meses, com implicações para a saúde ${ }^{1}$, apresenta como principais causas para a falência renal, as doenças de base, como diabetes mellitus e hipertensão arterial $^{2}$.

Além das causas patológicas, destaca-se a influência do processo natural de envelhecimento, que afeta todos os componentes do rim, levando a alterações na morfologia renal e a diminuição de sua função, que podem ser agravadas pela presença de comorbidades ${ }^{3}$. Neste contexto, estudos populacionais, em diferentes países, demonstram que a DRC atinge $7,2 \%$ de indivíduos acima de 30 anos e que esta prevalência aumenta, expressivamente, entre 23 e $36 \%$ em pessoas acima de 64 anos $^{4}$.

No Brasil, há inexistência de dados suficientes que demonstrem a amplitude da incidência e prevalência da DRC. Se a realidade norte-americana, em que cerca de $10 \%$ da população apresenta DRC, fosse tida como referência, a estimativa seria de aproximadamente 20 milhões de brasileiros estariam acometidos com a doença ${ }^{4}$.

Como a DRC, em geral, é silenciosa, tornase fundamental sua detecção precoce, bem como condutas terapêuticas apropriadas para o seu retardamento e sua progressão, a fim de diminuir a queda na qualidade de vida dos portadores da doença e os custos financeiros associados ${ }^{5}$.

Neste sentido, a educação nutricional proporciona o acesso a informações que trabalham questões subjetivas da alimentação que contribuem para a melhoria da qualidade de vida dos indivíduos. Uma proposta de intervenção nutricional concebida especificamente para um grupo de pacientes pode favorecer a adesão às orientações e retardar a progressão da DRC.

Assim, o presente estudo objetivou avaliar o impacto de um programa de intervenção nutricional sobre o estado nutricional e a qualidade de vida de idosos com DRC não dialítica.

\section{Métodos}

\section{Participantes}

Foi realizado estudo longitudinal com 64 idosos, de idade igual ou superior a 60 anos, de ambos os sexos, portadores de DRC estágio 3, que originaram uma coorte prospectiva acompanha- da por nove meses (de agosto de 2012 a maio de 2013), em uma Unidade Básica de Saúde (UBS), localizada no município de Diadema, São Paulo.

\section{Recrutamento e seleção}

Os idosos foram, inicialmente, selecionados por meio do HIPERDIA, um sistema que permite cadastrar e acompanhar os portadores de hipertensão arterial e/ou diabetes mellitus atendidos na rede ambulatorial do Sistema Único de Saúde (SUS) ${ }^{6}$.

Uma das informações fornecidas pelo HIPERDIA é o valor de creatinina sérica, empregado no cálculo para estimativa da taxa de filtração glomerular (TFG).

O estadiamento da DRC, para identificar os pacientes em estágio 3, partiu dos valores da TFG entre 30 e $59 \mathrm{~mL} / \mathrm{min} / 1,73 \mathrm{~m}^{2}$ estimada pela Equação de Cockcroft-Gault $t^{7,8}$.

Para o recrutamento dos participantes foram verificadas todas as fichas do HIPERDIA de 2010 a 2012, e selecionados os pacientes que atendiam aos critérios de inclusão: indivíduos com idade igual ou superior a 60 anos, portadores de DRC em estágio 3 .

Todos os idosos que estavam em conformidade com os critérios de inclusão foram convidados a participar da pesquisa, mediante documento entregue em domicílio pelo Agente Comunitário de Saúde do município, totalizando 146 indivíduos, dos quais 98 aceitaram participar e fizeram parte da amostragem inicial, tendo permanecido até o final do acompanhamento 64 deles.

Apenas após a aprovação pelo Comitê de Ética e Pesquisa (CEP), os idosos foram convidados e ocorreu o primeiro contato com a pesquisadora, quando tiveram a ciência dos riscos e dos benefícios da participação na pesquisa e dos critérios de exclusão: idoso portador de DRC em estágio 3, que deixasse de comparecer ao atendimento individual ou que tivesse frequência inferior a $75 \%$ nos encontros propostos. Aqueles que concordaram em participar, assinaram o Termo de Consentimento Livre e Esclarecido, atendendo aos aspectos éticos.

\section{Intervenção nutricional}

Durante a intervenção nutricional os idosos participaram de maneira coletiva e individual, sendo realizados quatro encontros (três coletivos e um individual). É importante destacar que como o idoso que faltasse no atendimento individual seria excluído do estudo, optou-se em con- 
tatar novamente os pacientes que faltaram, como uma espécie de "repescagem". Somente após este procedimento é que o programa teve continuidade para a próxima etapa, o que evitou muitas perdas ao longo do estudo.

As intervenções coletivas foram realizadas em três encontros, que duravam aproximadamente uma hora, com no máximo 15 idosos (Quadro 1).

Em relação ao conteúdo nutricional abordado nas intervenções coletivas, destacam-se: fracionamento das refeições ao longo do dia, ingestão de líquidos, conteúdo de potássio nos grupos alimentares das frutas, legumes e verduras e informações sobre o consumo moderado de proteínas, gorduras e sódio.

No encontro individual, após a primeira intervenção coletiva, foi entregue o plano alimentar individualizado. Para sua elaboração, foram consideradas informações obtidas no Recordatório de 24 horas, preferências e aversões alimentares e as recomendações nutricionais para a fase não dialítica da DRC (energia, carboidratos, proteínas, lipídeos, fibras e micronutrientes). Ao definir as metas nutricionais para cada paciente, os planos alimentares foram calculados no AVANUTRI, Software de Avaliação e Prescrição Nutricional, versão 4.0.

Ressalta-se que não houve uma avaliação específica para cada estratégia do programa de intervenção, sendo adotada, portanto, uma avaliação somativa, que permite medir o grau de alcance, ou de não alcance, dos resultados esperados $^{9}$. Para tanto, a intervenção nutricional foi avaliada ao final do processo, por meio dos resultados obtidos pela comparação entre as variáveis do estudo (antropométricas e de qualidade de vida) antes e após a intervenção.

\section{Perfil sociodemográfico, antropometria e qualidade de vida}

O registro dos dados sociodemográficos (sexo, idade, escolaridade e renda familiar mensal) ocorreu por meio de entrevista realizada pela nutricionista/pesquisadora, em momento anterior à intervenção nutricional.

As medidas antropométricas de peso, estatura e circunferência da cintura (CC) foram aferidas utilizando-se técnicas padronizadas, antes e após o programa de intervenção nutricional. O peso corporal foi aferido em balança antropométrica digital, com escala de 50g, sendo a carga máxima $200 \mathrm{~kg}$. Os idosos foram posicionados no centro do equipamento, com o mínimo de roupa possível, descalços, eretos, com os pés juntos e braços estendidos ao longo do corpo. Foram mantidos nessa posição até o valor do peso corporal estabilizar no visor da balança para a sua leitura ${ }^{10}$.

$\mathrm{Na}$ mensuração da estatura, com o uso do estadiômetro acoplado à balança com escala de $0,5 \mathrm{~cm}$ e altura máxima de $2 \mathrm{~m}$, os idosos se mantiveram descalços, com o peso distribuído entre

Quadro 1. Descrição das estratégias de intervenção coletiva realizadas com os idosos portadores de DRC não dialítica.

\begin{tabular}{|c|c|c|}
\hline Tema & Objetivos & Estratégia Pedagógica \\
\hline $\begin{array}{l}\text { Entendendo } \\
\text { o rim e suas } \\
\text { funções }\end{array}$ & $\begin{array}{l}\text { Apresentar o conceito e as funções dos rins, } \\
\text { evidenciando sua importância no equilíbrio } \\
\text { corporal. } \\
\text { - Expor a relação entre o envelhecimento e a } \\
\text { função renal. } \\
\text { - Introduzir a definição e classificação da DRC, } \\
\text { bem como as principais causas para o seu } \\
\text { desenvolvimento. }\end{array}$ & $\begin{array}{l}\text { Palestra: comunicação verbal entre } \\
\text { a pesquisadora e o grupo de idosos, } \\
\text { permitindo a oportunidade de } \\
\text { questionamento a qualquer momento. } \\
\text { Recurso: Data show. }\end{array}$ \\
\hline $\begin{array}{l}\text { Alimentação } \\
\text { para } \\
\text { pacientes } \\
\text { com DRC }\end{array}$ & $\begin{array}{l}\text { - Reforçar as orientações nutricionais para } \\
\text { pacientes com DRC na fase não dialítica. } \\
\text { - Discutir e refletir sobre as principais } \\
\text { dificuldades e facilidades em aderir ao plano } \\
\text { alimentar prescrito (encontro individual). }\end{array}$ & $\begin{array}{l}\text { Roda de conversa: criação de espaços } \\
\text { de diálogo, nos quais os participantes } \\
\text { puderam se expressar e, sobretudo, } \\
\text { escutar os outros e a si mesmos, } \\
\text { promovendo troca de experiências. }\end{array}$ \\
\hline $\begin{array}{l}\text { Tratamentos } \\
\text { da DRC }\end{array}$ & $\begin{array}{l}\text { Retomar a classificação da DRC e apresentar } \\
\text { os tratamentos realizados com a progressão } \\
\text { da doença para os estágios mais avançados } \\
\text { (tratamento dialítico). }\end{array}$ & $\begin{array}{l}\text { - Exposição oral: preleção verbal } \\
\text { sobre os tipos de tratamento na } \\
\text { DRC avançada, possibilitando aos } \\
\text { participantes sanar as dúvidas durante } \\
\text { a exposição do conteúdo. } \\
\text { - Recurso: Pôster. }\end{array}$ \\
\hline
\end{tabular}


os pés, calcanhares juntos, em postura ereta, com a cabeça livre de adereços e olhar voltado para frente em um ponto fixo na altura dos olhos ${ }^{10}$.

A partir do peso e da estatura, calculou-se o IMC, classificando o estado nutricional em: baixo peso, IMC $<23 \mathrm{~kg} / \mathrm{m}^{2}$; eutrofia, IMC $\geq 23 \mathrm{e}<$ $28 \mathrm{~kg} / \mathrm{m}^{2}$; sobrepeso, IMC $\geq 28 \mathrm{e}<30 \mathrm{~kg} / \mathrm{m}^{2} ; \mathrm{e}$, obesidade, IMC $\geq 30 \mathrm{~kg} / \mathrm{m}^{2}{ }^{11}$.

Para aferição da CC, com utilização de fita métrica de fibra de vidro inelástica, variação em milímetros, os idosos permaneceram em posição ereta, com os braços estendidos ao longo do corpo, pernas paralelas ligeiramente separadas e com o peso distribuído de maneira uniforme entre os pés. A fita foi posicionada horizontalmente no ponto médio entre a borda inferior da última costela e o topo da crista ilíaca, após o posicionamento foi solicitado que o idoso inspirasse e, em seguida, expirasse totalmente para a leitura da medida ${ }^{10,12}$.

Os pontos de corte adotados para classificação foram os estabelecidos pela Organização Mundial da Saúde (OMS) para adultos, uma vez que não há, ainda, valores específicos para idosos. Assim, valores de CC $\geq 80 \mathrm{~cm}$ para as mulheres, e $\geq 94 \mathrm{~cm}$ para os homens, foram considerados risco elevado para doenças cardiovasculares (DCV), enquanto que os valores $\geq 88 \mathrm{~cm}$ para mulheres, e $\geq 102 \mathrm{~cm}$ para homens, foram considerados risco muito elevado para o desenvolvimento de $\mathrm{DCV}^{13}$.

Para avaliar a percepção subjetiva da qualidade de vida foi utilizado o World Health Organization Quality of Life Questionnaire (WHOQOL-bref), desenvolvido pela OMS. Optou-se por sua utilização considerando que tem sido uma ferramenta útil para medir a qualidade de vida na população de doentes renais crônicos ${ }^{14}$. É um questionário composto por 26 questões, agrupadas em quatro domínios (físico, psicológico, social e ambiental) e duas de autopercepção, a primeira diz respeito a como o idoso avalia sua qualidade de vida enquanto a segunda refere-se à satisfação quanto seu estado de saúde ${ }^{15}$.

A aplicação do WHOQOL-bref ocorreu em dois momentos, antes e após a intervenção nutricional, sob a forma de entrevista, considerando a possibilidade dos indivíduos apresentarem queda da acuidade visual e baixo nível instrucional.

A pontuação para cada domínio e para cada questão varia de zero a cinco, sendo que não há pontos de corte para melhor ou pior qualidade de vida. Assim, supõe-se que melhor qualidade de vida seja indicada por valores mais próximos a 100, e pior qualidade de vida, por valores mais próximos a zero.

\section{Análise de dados}

Para análise estatística utilizou-se o software Statistical Package for Social Science (SPSS), versão 21, adotando nível de significância de 5\% (p $\leq 0,05$ ). Para comparação das médias do IMC, da CC, da pontuação dos domínios, das questões de autopercepção e da pontuação geral do WHOQOL-bref, antes e após o programa de intervenção nutricional, foi utilizado o teste t de Student para grupos pareados.

\section{Resultados}

Os idosos estudados apresentaram idade média de 73,95 \pm 7,84 anos, com variação entre $60 \mathrm{e}$ 91 anos e distribuição semelhante entre aqueles com idade $<75 \mathrm{e} \geq 75$ anos. A amostra foi constituída, predominantemente, por idosos do sexo feminino $(65,6 \%)$, que não concluíram o ensino fundamental ou não apresentaram escolaridade $(92,2 \%)$ e que possuíam renda familiar mensal de até três salários mínimos (76,6\%) (Tabela 1).

Em relação ao estado nutricional, observou-se maior frequência de idosos eutróficos. No entanto, $21,9 \%$ apresentaram baixo peso e $32,8 \%$ excesso de peso (sobrepeso ou obesidade). Quando estratificado por sexo, o excesso de peso foi mais evidente nas mulheres do que nos homens. Ao avaliar o risco para o desenvolvimento de DCV pela CC, $62,6 \%$ dos idosos apresentaram risco cardiovascular elevado ou muito elevado,

Tabela 1. Distribuição de idosos portadores de DRC não dialítica, segundo características sociodemográficas.

\begin{tabular}{lcc}
\hline \multicolumn{1}{c}{ Variáveis sociodemográficas } & n & $\%$ \\
\hline Sexo & & \\
$\quad$ Masculino & 22 & 34,4 \\
$\quad$ Feminino & 42 & 65,6 \\
Grupo etário & & \\
$\quad<75$ anos & 33 & 51,6 \\
$\quad \geq 75$ anos & 31 & 48,4 \\
Escolaridade & & \\
$\quad$ Sem escolaridade & 20 & 31,3 \\
$\quad$ Ensino Fundamental Incompleto & 39 & 60,9 \\
$\quad$ Ensino Fundamental Completo & 05 & 7,8 \\
Renda familiar mensal & & \\
$\quad$ Até 3 salários mínimos & 49 & 76,6 \\
$\quad \geq 3$ salários mínimos & 15 & 23,4 \\
\hline
\end{tabular}


sendo que nos homens o risco cardiovascular muito elevado foi mais frequente (Tabela 2).

A média e o desvio padrão do IMC e da CC dos idosos estudados de acordo com o sexo, antes e após a intervenção nutricional, são apresentados na Tabela 3. Sendo assim, a partir da comparação entre as médias do IMC, observou-se redução estatisticamente significante, em ambos os sexos, após o programa de intervenção nutricional. Para a CC verificou-se que, tanto para o sexo feminino quanto para o masculino, houve diminuição estatisticamente significante entre as médias, sen- do que nas mulheres, apesar dessa redução, ainda prevaleceu o risco cardiovascular elevado.

Quanto à qualidade de vida, avaliada pelo WHOQOL-bref, observou-se que a maior pontuação atribuída pelos idosos foi para o domínio social, que se refere à satisfação com suas relações pessoais e sexuais, e a menor pontuação para o domínio ambiental, que está relacionado a quanto o idoso se sente seguro, tem oportunidades de lazer, condições de moradia, acesso aos serviços de saúde, meio de transporte e condições financeiras (Tabela 4).

Tabela 2. Distribuição de idosos portadores de DRC não dialítica, segundo estado nutricional e risco cardiovascular.

\begin{tabular}{clccc}
\hline Condições de saúde & Classificação & Masculino $(\mathbf{n}=\mathbf{2 2})$ & Feminino $(\mathbf{n}=\mathbf{4 2})$ & Total $(\mathbf{n}=\mathbf{6 4})$ \\
\hline Estado nutricional & Baixo peso & $6(27,3 \%)$ & $8(19,0 \%)$ & $14(\mathbf{2 1 , 9 \% )}$ \\
& Eutrofia & $11(50,0 \%)$ & $18(42,5 \%)$ & $29(45,3 \%)$ \\
& Sobrepeso & $1(4,5 \%)$ & $7(16,7 \%)$ & $8(12,5 \%)$ \\
& Obesidade & $4(18,5 \%)$ & $9(21,4 \%)$ & $13(20,3 \%)$ \\
\multirow{2}{*}{ Risco Cardiovascular } & Sem risco & $6(27,3 \%)$ & $18(42,9 \%)$ & $24(37,5 \%)$ \\
& Elevado & $3(13,6 \%)$ & $17(40,5 \%)$ & $20(\mathbf{3 1 , 3} \%)$ \\
& Muito elevado & $13(59,1 \%)$ & $7(16,7 \%)$ & $20(\mathbf{3 1 , 3 \% )}$ \\
\hline
\end{tabular}

Tabela 3. Média e desvio padrão do IMC e da CC de idosos portadores de DRC não dialítica, segundo o sexo, pré e pós-intervenção.

\begin{tabular}{ccccc}
\hline \multirow{2}{*}{ Sexo } & & Pré & Pós & \multirow{2}{*}{ p } \\
\cline { 3 - 4 } & & Média \pm DP & Média \pm DP & \\
\hline Feminino $(\mathrm{n}=42)$ & IMC & $26,75 \pm 3,72$ & $26,23 \pm 3,67$ & $\mathbf{0 , 0 1 5}$ \\
& CC & $85,68 \pm 8,04$ & $83,62 \pm 8,00$ & $\mathbf{0 , 0 0 3}$ \\
Masculino $(\mathrm{n}=22)$ & IMC & $25,59 \pm 3,82$ & $24,99 \pm 3,76$ & $\mathbf{0 , 0 0 1}$ \\
& CC & $89,71 \pm 10,70$ & $87,91 \pm 10,96$ & $\mathbf{0 , 0 0 1}$ \\
\hline
\end{tabular}

Tabela 4. Média e desvio padrão da pontuação dos domínios, das questões de autopercepção e da pontuação geral do WHOQOL-bref de idosos portadores de DRC não dialítica, pré e pós-intervenção.

\begin{tabular}{lccc}
\hline \multirow{1}{*}{\multicolumn{1}{c}{ Variáveis }} & Pré & Pós & p \\
\cline { 2 - 3 } & Média \pm DP & Média \pm DP & 0,845 \\
\hline Domínio físico & $59,43 \pm 13,67$ & $59,77 \pm 14,39$ & 0,292 \\
Domínio psicológico & $61,07 \pm 13,01$ & $62,70 \pm 15,31$ & 0,682 \\
Domínio social & $70,83 \pm 12,24$ & $71,61 \pm 12,75$ & 0,729 \\
Domínio ambiental & $56,01 \pm 10,10$ & $55,42 \pm 10,64$ & 0,311 \\
Autopercepção 1 & $64,84 \pm 19,27$ & $62,11 \pm 18,36$ & $\mathbf{0 , 0 3 5}$ \\
Autopercepção 2 & $56,64 \pm 22,82$ & $62,89 \pm 20,89$ & 0,633 \\
Pontuação geral & $60,17 \pm 8,76$ & $60,68 \pm 9,61$ & \\
\hline
\end{tabular}


Verificou-se que não houve ganhos e nem perdas após a intervenção nutricional: para todos os domínios, para a questão 1 de autopercepção e para a pontuação geral. No entanto, houve um aumento estatisticamente significante da satisfação dos idosos sobre seu estado de saúde após a intervenção, demonstrado pela diferença entre as médias da segunda questão de autopercepção que avalia "Quão satisfeito(a) você está com a sua saúde?” (Tabela 4).

\section{Discussão}

O conhecimento do perfil dos pacientes com DRC e o tratamento nos estágios iniciais, com a adoção de condutas individualizadas e adequadas pode retardar a evolução da doença e evitar complicações, melhorando a qualidade de vida dos pacientes e reduzindo os custos com tratamento ${ }^{16}$. Dessa forma, estratégias capazes de melhorar a adesão às orientações nutricionais, como o desenvolvimento de ações de educação nutricional, abrangendo planejamento, implementação e avaliação dessas ações, são importantes e merecem ser desenvolvidas ${ }^{17}$. Neste contexto, este estudo se propôs a avaliar o impacto de um programa de intervenção nutricional realizado com idosos portadores de DRC não dialítica, por meio da comparação das variáveis antropométricas e de qualidade de vida, antes e após a intervenção.

Em relação ao estado nutricional, uma pesquisa transversal realizada por Martin et al..$^{18} \mathrm{em}$ São Paulo, com idosos de ambos os sexos, encontrou resultados semelhantes ao do presente estudo quanto à distribuição dos idosos segundo o IMC, sendo que mais da metade da amostra apresentou eutrofia, $14,3 \%$ desnutrição e $21,4 \%$ excesso de peso. Ao estudar idosos portadores de DRC, Rembold et al. ${ }^{16}$ verificaram que $6,9 \%$ dos pacientes estavam abaixo do peso e $54,1 \%$ com excesso, evidenciando que tanto os idosos com diagnóstico de DRC quanto os que não tem a doença apresentam frequência relevante de inadequação do estado nutricional.

Os pacientes portadores de DRC estão propensos a apresentar desnutrição, quadro marcador de mau prognóstico, por isso, a avaliação do estado nutricional tem o objetivo identificar os indivíduos em risco ${ }^{19}$. Embora a desnutrição seja comum em pacientes com problemas renais, há, relativamente, poucos estudos abordando sua prevalência em pacientes na fase não dialítica ${ }^{20}$. Além disso, há variação na prevalência da desnutrição dependendo dos critérios utilizados para o diagnóstico (bioquímicos: albumina, creatinina; antropométricos: perímetro de braço, IMC; e, nutricionais: avaliação subjetiva global ${ }^{21}$. Dessa forma, a definição da desnutrição com base na hipoalbuminemia tem sido relatada com prevalência de 20 a 45\%, já a desnutrição com base na avaliação subjetiva global tem mostrado prevalência de 18 a $20 \%{ }^{20}$. As prevalências de desnutrição citadas concordam com o resultado de baixo peso encontrado no presente estudo (21,9\%), verificada por meio de indicador antropométrico (IMC). A importância em diagnosticar pacientes renais crônicos com desnutrição está relacionada à sua influência no risco de infecção, hospitalização e mortalidade, inflamação e diminuição da qualidade de vida ${ }^{21}$.

Apesar da elevada prevalência de desnutrição encontrada em pacientes com DRC, tem-se observado que o sobrepeso e a obesidade também são distúrbios nutricionais frequentes nessa população ${ }^{8}$. Em torno de 50 a $60 \%$ dos pacientes na fase não dialítica apresentam sobrepeso e obesidade avaliados pelo $\mathrm{IMC}^{22}$. No presente estudo, quando estratificado por sexo, o excesso de peso foi mais evidente nas mulheres $(38,1 \%)$ do que nos homens $(23 \%)$. Resultado também observado no estudo "Saúde, bem-estar e envelhecimento" (SABE), coordenado pela Organização Pan-Americana da Saúde (OPAS), com idosos residentes na área urbana do município de São Paulo, em que a maior proporção de sobrepeso e obesidade foi verificada para o sexo feminino (40,5\%) em comparação ao masculino $(21,7 \%)$. Esse achado parece refletir, dentre outros fatores, maior quantidade de gordura corporal, uma vez que mulheres apresentam valores de massa corporal e estatura inferiores aos homens. Sabe-se que isso ocorre em todas as idades e se dá, principalmente, devido à gordura, que no sexo feminino é, aproximadamente, quatro vezes superior à do sexo masculino ${ }^{23}$.

Esses resultados mostram que não há um perfil único no estado nutricional do paciente com DRC e, por isso, se faz necessário atenção tanto para o paciente com desnutrição quanto para aquele com excesso de peso, uma vez que a obesidade é um importante fator de risco para a hipertensão e a diabetes tipo 2, e quando presentes simultaneamente, agravam a lesão renal ${ }^{24}$. Além de estar associada ao desenvolvimento da DRC, também tem papel na sua progressão para os estágios mais avançados e na mortalidade relacionada à doença renal ${ }^{25}$. A maioria dos tratamentos e abordagens para reduzir a lesão renal em pacientes obesos foca no gerenciamento de 
fatores de risco associados, como hipertensão, diabetes e hiperlipidemia, utilizando estratégias como aconselhamento nutricional, interferência farmacológica e, em alguns casos, cirúrgica ${ }^{24}$. Nesse sentido, para pacientes com peso inicial normal, o ganho de peso deve ser evitado e a perda de peso é recomendada para aqueles que estão acima do peso, em especial, pela contribuição de seus benefícios para o controle da pressão arterial e da glicemia ${ }^{25}$.

O risco para o desenvolvimento de DCV pela $\mathrm{CC}$, à semelhança do estudo realizado por Sanches et al. ${ }^{26}$ com 122 adultos portadores de DRC não dialítica com idade entre 27 e 79 anos, mostrou que os homens apresentam uma área significativamente maior de gordura visceral quando comparados as mulheres.

Esses resultados chamam a atenção para o fato de a gordura abdominal ser um fator que favorece a ocorrência de eventos cardiovasculares. Destaca-se, ainda, que a prevalência de DCV nos pacientes com DRC é mais elevada que na população geral, inclusive naqueles que se encontram na fase pré-dialítica, e constitui a principal causa de morbidade e mortalidade nesta população, especialmente naqueles em diálise ${ }^{27}$.

Ao avaliar o impacto da intervenção nutricional observou-se redução estatisticamente significante entre as médias do IMC e da CC, em ambos os sexos, após a intervenção.

Em ensaio clínico randomizado, controlado e prospectivo com pacientes portadores de DRC não dialítica, com idade média de 63,4 \pm 11,0 anos, verificou-se resultado semelhante a da presente pesquisa, em que houve redução estatisticamente significante do IMC ao final do acompanhamento em ambos os grupos (intervenção e controle). Para a CC, houve diminuição estatisticamente significante para os homens no grupo intervenção e para as mulheres no grupo controle, sendo observada redução em ambos os grupos ${ }^{17}$. Esses resultados são favoráveis, considerando os benefícios relacionados à redução de peso corporal e da CC no curso da doença renal, visto que o excesso de peso e os eventos cardiovasculares são tidos como uma das principais causas de morbidade e mortalidade em pacientes com DRC.

Wang et al. ${ }^{28}$ estudaram a influência da intervenção dietética e do exercício físico em 38 pacientes obesos com DRC. Observaram após um programa de intervenção de estilo de vida de dois meses que os pacientes com controle do peso corporal apresentaram melhora significativa no controle da pressão arterial, no perfil lipídico, no nível de creatinina sérica, na taxa de filtração glomerular estimada e na proteinúria. Esses resultados evidenciam que a combinação da intervenção dietética e exercício físico associou-se à melhora nos fatores de risco cardiovascular e perfil renal em pacientes obesos com DRC.

É importante mencionar que a redução do IMC deve ser avaliada individualmente e com cautela nos pacientes renais crônicos, em especial idosos, considerando os prejuízos relacionados à desnutrição. Ricardo et al. ${ }^{29}$ avaliaram a associação entre estilo de vida saudável e menor risco de morte em participantes do Third National Health and Nutrition Examination Survey (NHANES III) portadores de DRC. Os autores relatam um aumento do risco de mortalidade de $30 \%$ entre os participantes com IMC de 18,5 a $22 \mathrm{~kg} / \mathrm{m}^{2}$, sugerindo que evitar o baixo IMC pode melhorar a sobrevida dos pacientes. Embora a obesidade geral possa conferir um papel protetor em eventos catabólicos que acometem os portadores de DRC, por outro lado, a obesidade abdominal é um fator de risco para menor sobrevida nesses pacientes ${ }^{30}$, demonstrando a importância da redução da CC e do IMC. No entanto, a associação entre IMC e seus efeitos tem sido menos estudada em pacientes com DRC não dialítica, o que sugere a realização de pesquisas futuras para avaliar melhor essa relação, bem como para determinar o que constitui um IMC ideal para essa população ${ }^{29}$.

Apesar da utilização do IMC como ferramenta para avaliação do estado nutricional em estudos epidemiológicos, na prática clínica o IMC pode não ser uma ferramenta muito precisa na monitorização de gordura corporal ou estado nutricional, particularmente na DRC. Isso devido a algumas limitações como a não diferenciação dos compartimentos corporais, como massa gordurosa e massa magra - músculos ${ }^{31,32}$ e a possibilidade de "mascarar" o estado nutricional do paciente em situações de retenção hídrica, comuns nessa população ${ }^{33}$, especialmente nas fases mais avançadas da doença. Neste estudo não foi realizada a avaliação de edema, no entanto, é importante destacar que os idosos não se encontravam em fase avançada da DRC.

Assim, em razão da importância da composição corporal no prognóstico dos pacientes com DRC, a utilização do IMC e da CC na prática clínica não deve ocorrer isoladamente, mas associada a outros métodos e ferramentas de avaliação do estado nutricional e da composição corporal, a fim de estabelecer um diagnóstico nutricional adequado $^{34}$. 
Quanto ao impacto do programa em relação à qualidade de vida, constatou-se um aumento estatisticamente significante da satisfação dos idosos sobre seu estado de saúde após a intervenção, o que pode ser considerado positivo, visto que diante da situação em que os pacientes renais crônicos encontram-se, em que a cura não é uma meta atingível, melhorar a satisfação do paciente com seu tratamento e seu bem-estar geral deveria tornar-se um dos objetivos primários ${ }^{14}$.

Campbell et al. ${ }^{35}$ avaliaram o impacto de uma intervenção nutricional na qualidade de vida de pacientes com DRC na pré-diálise, divididos em grupo intervenção e controle. Por meio do uso da escala Kidney Disease Quality of Life Short Form, combinando o Short Form-36 (SF-36) com o módulo específico para doença renal, os autores observaram diferença estatisticamente significante na média da pontuação de sintomas da doença renal, funcionamento cognitivo e vitalidade, em favor da intervenção.

Santos et al. ${ }^{36}$ avaliaram o impacto do acompanhamento interdisciplinar na qualidade de vida de pacientes com DRC na fase pré-dialítica. Foi verificado que após um ano de acompanhamento o grupo que recebeu intervenção interdisciplinar obteve melhora estatisticamente significante em cinco dos oito domínios avaliados pela escala Medical Outcomes Study Questionaire 36 Item Short Form Health Survey (SF-36). Em contrapartida, no grupo controle (atendimento convencional) não se observou melhora em nenhum dos parâmetros de qualidade de vida avaliados.

$\mathrm{Na}$ literatura, poucos estudos avaliaram a qualidade de vida de pacientes na fase não dialítica. Além disso, grande parte dos estudos foca a avaliação de parâmetros físicos e laboratoriais, em detrimento de parâmetros psicológicos e de qualidade de vida ${ }^{36}$. Os achados do presente estudo, bem como das pesquisas citadas, mesmo utilizando diferentes instrumentos para avaliar a qualidade de vida, apontam para a importância de intervenções precoces, nutricionais e interdisciplinares, em pacientes ainda na fase não dialítica, tendo em vista a melhora da qualidade de vida dessa população, desde o diagnóstico da DRC até a entrada em terapia renal substitutiva.

Neste contexto, destaca-se que, diante da complexidade da DRC e dos problemas clínicos próprios do envelhecimento, o modelo de atendimento interdisciplinar, por possibilitar uma avaliação multidimensional do paciente, surge como a melhor forma de tratar os pacientes idosos portadores de $\mathrm{DRC}^{37}$, incluindo a atuação do nutricionista em programas de educação alimentar e nutricional.
Cabe mencionar que devido às limitações do presente estudo, quanto à amostra ser não probabilística e não ter a presença de um grupo controle, os resultados encontrados devem ser analisados com cautela, não podendo ser generalizados para a população de idosos portadores de DRC. Adicionalmente, pode-se referir algumas limitações quanto à utilização do Recordatório de 24 horas para avaliação do consumo alimentar, considerando que a qualidade da informação coletada depende, entre outros fatores, da memória, da cooperação do paciente e da idade. Sendo que esta última influencia a habilidade do indivíduo em recordar seu consumo de alimentos de forma precisa, sobretudo nas idades extremas, quando se requer que uma pessoa responsável relate a informação ${ }^{38}$.

Por fim, sugere-se a realização de pesquisas randomizadas, controladas e prospectivas com foco em intervenções nutricionais educativas com idosos portadores de DRC, abrangendo planejamento, implementação e avaliação dessas ações.

\section{Conclusões}

A partir dos resultados obtidos pode-se concluir que o programa de intervenção nutricional proporcionou impacto positivo no estado nutricional, diminuindo o risco de desenvolvimento de doenças cardiovasculares e promoveu aumento da satisfação dos idosos em relação ao estado de saúde, repercutindo na melhora da qualidade de vida. Isso se deve, provavelmente, à intervenção nutricional realizada que disponibilizou informações em relação à doença e aos cuidados com a alimentação, aumentando o comprometimento do paciente com o seu tratamento.

Apesar das limitações, considerando a literatura escassa sobre intervenção nutricional em pacientes com DRC antes da terapia dialítica, especialmente em idosos, este estudo tem muito a contribuir para os profissionais da área quanto à prestação do cuidado nutricional baseado em práticas educativas, visando à adesão às orientações dietéticas pelos pacientes.

Ainda, diante dos benefícios decorrentes das ações educativas desenvolvidas durante a intervenção nutricional, destaca-se a necessidade de instituir políticas públicas que estabeleçam medidas de educação alimentar e nutricional na Atenção Básica, com vistas às ações eficazes e de baixo custo. $\mathrm{O}$ foco dessas ações deve ser a prevenção da DRC em pacientes hipertensos e diabéticos, principais acometidos pela doença renal, 
além de ações que promovam o retardo da progressão da DRC, instituídas em estágios precoces da doença, a fim de melhorar a qualidade de vida dos pacientes e reduzir os custos financeiros com tratamentos dialíticos.

\section{Colaboradores}

FG Magalhães trabalhou na concepção, coleta e interpretação dos dados e na redação final, RMM Goulart, na concepção, revisão crítica e aprovação da versão a ser publicada e LC Prearo, na análise dos dados e aprovação da versão a ser publicada.

\section{Referências}

1. Kidney Disease: Improving Global Outcomes (KDIGO) CKD Work Group. KDIGO 2012 Clinical Practice Guideline for the Evaluation and Management of Chronic Kidney Disease. Kidney Int 2013; 3(1):1150.

2. Bastos MG, Bregman R, Kirsztajn GM. Doença renal crônica: frequente e grave, mas também prevenível e tratável. Rev Assoc Med Bras 2010; 56(2):248-253.

3. Scapini KB, Leguisamo CP, Filho HT, Tagliaro ML, Bertolin TE. Estresse oxidativo, envelhecimento renal e doença renal crônica terminal. Estud interdiscipl Envelhec 2010; 15(2):279-293.

4. Abreu PF. Epidemiologia. In: Cuppari L, Avesani CM, Kamimura MA. Nutrição na doença renal crônica. Barueri: Manole; 2013. p. 3-31.

5. Romão Júnior JE. Doença renal crônica: definição, epidemiologia e classificação. J Bras Nefrol 2004; 3(3):1-3.

6. Brasil. Ministério da Saúde (MS). Secretaria de Atenção à Saúde. Departamento de Atenção Básica. SISHIPERDIA: Apresentação. 2014. [acessado 2014 Jul 21]. Disponível em: http://hiperdia.datasus.gov.br/.

7. Brasil. Ministério da Saúde (MS). Prevenção clínica de doenças cardiovasculares, cerebrovasculares e renais. Brasília: MS; 2006.

8. National Kidney Foundation. K/DOQI Clinical Practice Guidelines for Chronic Kidney Disease: Evaluation, Classification and Stratification. Am J Kidney Dis 2002; 39(Supl. 1):1-266.

9. Boog MCF. Educação em nutrição: integrando experiências. Campinas: Komedi; 2013.

10. Brasil. Ministério da Saúde (MS). Orientações para a coleta e análise de dados antropométricos em serviços de saúde: Norma Técnica do Sistema de Vigilância Alimentar e Nutricional - SISVAN. Brasília: MS; 2011.

11. Organização Pan-Americana da Saúde (OPAS). Encuesta Multicêntrica: salud, bien estar y envejecimiento $(S A B E)$ en América Latina y el Caribe. Washington: OPAS; 2001.

12. World Health Organization (WHO). Waist circumference and waist-hip ratio: report of a WHO expert consultation. Geneva: WHO; 2008.

13. World Health Organization (WHO). Obesity: Preventing and managing the global epidemic: report of a WHO consultation. Geneva: WHO; 2000. 
14. Cruz LN, Polanczyk CA, Fleck MPA. Qualidade de vida em insuficiência renal crônica. In: Fleck MPA, organizador. A avaliação de qualidade de vida: guia para profissionais da saúde. Porto Alegre: Artmed; 2008. p. 208-218.

15. Chachamovich E, Fleck MPA. Desenvolvimento do WHOQOL-bref. In: Fleck MPA, organizador. A avaliação de qualidade de vida: guia para profissionais da saúde. Porto Alegre: Artmed; 2008. p. 74-82.

16. Rembold SM, Santos SLS, Vieira GB, Barros MS, Lugon JR. Perfil do doente renal crônico no ambulatório multidisciplinar de um hospital universitário. Acta Paul Enferm 2009; 22(n. esp. 1):501-504.

17. Barreto JGP. Avaliação do impacto de um programa de educação nutricional sobre a adesão à dieta hipoproteica em pacientes com doença renal crônica em tratamento conservador [dissertação]. Rio de Janeiro: Universidade do Estado do Rio de Janeiro; 2011.

18. Martin FG, Nebuloni CC, Najas MS. Correlação entre estado nutricional e força de preensão palmar em idosos. Rev Bras Geriatr Gerontol 2012; 15(3):493-504.

19. Daltrozo JB, Spillere A, Fraga CM. Avaliação do estado nutricional e do perfil clínico-epidemiológico dos pacientes em tratamento hemodialítico no Hospital São José de Criciúma e na Nefroclínica-Criciúma. ACM Arquivos Catarinenses de Medicina 2010; 39(4):12-17.

20. Carrero JJ, Cozzolino M. Nutritional therapy, phosphate control and renal protection. Nephron Clin Pract 2014; 126(1):1-7.

21. Gracia-Iguacel C, González-Parra E, Barril-Cuadrado G, Sánchez R, Egido J, Ortiz-Arduán A, Carrero JJ. Defining protein-energy wasting syndrome in chronic kidney disease: prevalence and clinical implications. Nefrologia 2014; 34(4):507-519.

22. Cuppari L, Kamimura MA. Avaliação nutricional na doença renal crônica: desafios na prática clínica. J Bras Nefrol 2009; 31(1):28-35.

23. Lebrão ML, Laurenti R. Saúde, bem-estar e envelhecimento: o estudo SABE no Município de São Paulo. Rev Bras Epidemiol 2005; 8(2):127-141.

24. Felizardo RJF, Silva MB, Aguiar CF, Câmara NOS. Obesity in kidney disease: A heavyweight oponente. World J Nephrol 2014; 3(3):50-63.

25. James MT, Hemmelgarn BR, Tonlelli M. Early recognition and prevention of chronic kidney disease. Lancet 2010; 375(9722):1296-1309.

26. Sanches FM, Avesani CM, Kamimura MA, Lemos MM, Axelsson J, Vasselai P, Draibe SA, Cuppari L. Waist circumference and visceral fat in CKD: a cross-sectional study. Am J Kidney Dis 2008; 52(1):66-73.

27. Ammirati AL, Canziani MEF. Fatores de risco da doença cardiovascular nos pacientes com doença renal crônica. J Bras Nefrol 2009; 31(Supl. 1):43-48.
28. Wang YL, Shu KH, Yang MF, Yang WC, Wu MJ, Lin TM, $\mathrm{Chen} \mathrm{CH}$. The impact of body weight management in chronic kidney disease patients with obesity. J Ren Nutr 2013; 23(5):372-379.

29. Ricardo AC, Madero M, Yang W, Anderson C, Menezes M, Fischer MJ, Turyk M, Daviglus ML, Lash JP. Adherence to a healthy lifestyle and all-cause mortality in CKD. Clin J Am Soc Nephrol 2013; 8(4):602-609.

30. Kamimura MA. Obesidade. In: Cuppari L, Avesani CM, Kamimura MA, organizadores. Nutrição na doença renal crônica. Barueri: Manole; 2013. p. 351.

31. Carrero JJ, Avesani CM. Pros and cons of body mass Index as a nutritional and risk assessment tool in dialysis patients. Semin Dial 2015; 28(1):48-58.

32. Tavares EL, Santos DM, Ferreira AA, Menezes MFG. Avaliação nutricional de idosos: desafios da atualidade. Rev Bras Geriatr Gerontol 2015; 18(3):643-650.

33. Kamimura MA. Antropometria e composição corporal. In: Cuppari L, Avesani CM, Kamimura MA, organizadores. Nutrição na doença renal crônica. Barueri: Manole; 2013. p. 137.

34. Magalhães FG, Goulart RMM. Doença renal crônica e tratamento em idosos: uma revisão integrativa. Rev Bras Geriatr Gerontol 2015; 18(3):679-692.

35. Campbell KL, Ash S, Bauer JD. The impact of nutrition intervention on quality of life in pre-dialysis chronic kidney disease patients. Clin Nutr 2008; 27(4):537-544.

36. Santos FR, Filgueiras MST, Chaoubah A, Bastos MG, Paula, RB. Efeitos da abordagem interdisciplinar na qualidade de vida e em parâmetros laboratoriais de pacientes com doença renal crônica. Rev Psiquiatr Clín 2008; 35(3):87-95.

37. Bastos MG, Oliveira DCQ, Kirsztajn GM. Doença renal crônica no paciente idoso. Rev HCPA 2011; 31(1):5265.

38. Fisberg RM, Marchioni DML, Colucci ACA. Avaliação do consumo alimentar e da ingestão de nutrientes na prática clínica. Arq Bras Endocrinol Metab 2009; 53(5):617-624.

Artigo apresentado em 29/02/2016

Aprovado em 05/09/2016

Versão final apresentada em 07/09/2016 